\title{
ACE: Asynchronous and Concurrent Execution of Complex Smart Contracts
}

\author{
Karl Wüst \\ Department of Computer Science \\ ETH Zurich \\ karl.wuest@inf.ethz.ch
}

\author{
Sinisa Matetic \\ Department of Computer Science \\ ETH Zurich \\ sinisa.matetic@inf.ethz.ch
}

\author{
Silvan Egli \\ Department of Computer Science \\ ETH Zurich \\ siegli@student.ethz.ch
}

\author{
Kari Kostiainen \\ Department of Computer Science \\ ETH Zurich \\ kari.kostiainen@inf.ethz.ch
}

\author{
Srdjan Capkun \\ Department of Computer Science \\ ETH Zurich \\ srdjan.capkun@inf.ethz.ch
}

\begin{abstract}
Smart contracts are programmable, decentralized and transparent financial applications. Because smart contract platforms typically support Turing-complete programming languages, such systems are often said to enable arbitrary applications. However, the current permissionless smart contract systems impose heavy restrictions on the types of computations that can be implemented. For example, the globally-replicated and sequential execution model of Ethereum requires low gas limits that make many computations infeasible.

In this paper, we propose a novel system called ACE whose main goal is to enable more complex smart contracts on permissionless blockchains. ACE is based on an off-chain execution model where the contract issuers appoint a set of service providers to execute the contract code independent from the consensus layer. The primary advantage of ACE over previous solutions is that it allows one contract to safely call another contract that is executed by a different set of service providers. Thus, ACE is the first solution to enable offchain execution of interactive smart contracts with flexible trust assumptions. Our evaluation shows that ACE enables several orders of magnitude more complex smart contracts than standard Ethereum.
\end{abstract}

\section{CCS CONCEPTS}

- Security and privacy $\rightarrow$ Distributed systems security.

\section{KEYWORDS}

blockchain; smart contracts; scalability;

\section{ACM Reference Format:}

Karl Wüst, Sinisa Matetic, Silvan Egli, Kari Kostiainen, and Srdjan Capkun. 2020. ACE: Asynchronous and Concurrent Execution of Complex Smart Contracts. In Proceedings of the 2020 ACM SIGSAC Conference on Computer and Communications Security (CCS '20), November 9-13, 2020, Virtual Event, USA. ACM, New York, NY, USA, 14 pages. https://doi.org/10.1145/3372297.3417243

Permission to make digital or hard copies of part or all of this work for personal or classroom use is granted without fee provided that copies are not made or distributed for profit or commercial advantage and that copies bear this notice and the full citation on the first page. Copyrights for third-party components of this work must be honored. For all other uses, contact the owner/author(s).

CCS '20, November 9-13, 2020, Virtual Event, USA

(C) 2020 Copyright held by the owner/author(s)

ACM ISBN 978-1-4503-7089-9/20/11.

https://doi.org/10.1145/3372297.3417243

\section{INTRODUCTION}

After Bitcoin [23] became the first permissionless cryptocurrency and popularized Blockchain technology, Ethereum [31] extended this concept to smart contract, which are programs that allow contract participants to load blockchain-based currency to a contractcontrolled account. The contract's code defines rules and conditions under which its funds will then be transferred out of the contract.

It is commonly argued that smart contracts provide significant advantages over traditional financial instruments. One advantage is their generality: since smart contracts are programmable, they should enable arbitrary financial applications on blockchains. The second is improved transparency: smart contract code and execution is verifiable by anyone from the public blockchain. And the third is strong liveness: contract execution is not controlled by one or few entities, but rather by a large permissionless system.

While these properties are attractive for building novel financial applications, current smart contract systems fail to realize them fully. For example, Ethereum, in principle, allows arbitrary contracts through a Turing complete language, but in practice it heavily limits contract complexity. Such limits are necessary, because Ethereum is based on a sequential and globally-replicated execution model, where every miner should execute all contract calls of the latest block before finding the next. Due to this high degree of replication, the computational complexity of contracts is inherently limited.

To prevent excessive delays, Ethereum uses a metric called gas to measure execution complexity. If a contract call surpasses a specified limit, its execution is aborted which effectively limits the types of computation that can be implemented. For example, the simple task of sorting 256 integers with selection sort requires $17 \mathrm{M}$ gas while the current limit for each block is $8 \mathrm{M}$. Quick sort hits the limit after 2,000 elements. More complex applications, such as decentralized blockchain oracles [28], quickly become infeasible.

Our main goal is to increase these per-block execution limits and to enable safe execution of more complex smart contracts for permissionless blockchain systems like Ethereum, while maintaining transparency and good liveness.

Previous work. Because Ethereum's execution model is both expensive and slow, recent research has explored alternative ways to execute contracts on permissionless blockchains.

YODA [10] proposes a randomly-sampled model that supports asynchronous execution of contract calls. For each contract call, a 
subset of miners is chosen randomly to execute the contract code independently of the mining process and return the results in form of a new transaction. Arbitrum [17] suggests a model where the contract creator appoints a small set of verifiers who should check execution integrity off-chain. Thus, Arbitrum replaces the sequential and globally-replicated execution model of Ethereum with one where different contracts can be executed (and thus verified) asynchronously by only few parties who may have an interest in the contract's integrity. Ekiden [8] uses Trusted Execution Environments (TEEs), namely SGX enclaves, to execute smart contracts. The main motivation of Ekiden is to enable confidential contracts, rather than complex ones, but since execution is decoupled from consensus, Ekiden can serve this purpose as well.

The above solutions execute contract calls in separate partitions, but none of the above works addresses safe and concurrent execution of transactions that cross partitions, i.e., cases where a contract executed in one partition calls a contract in another partition.

Many current Ethereum contracts call other contracts and change their state. Executing such contracts without concurrency control can leave the contracts in an inconsistent state, as is well-known from distributed databases [3]. Thus, none of the previous solutions enable safe execution of many current Ethereum contracts.

Our solution. In this paper, we propose a novel system that we call ACE (for Asynchronous and Concurrent Execution of Complex Smart Contracts). ACE combines elements from previous systems with an efficient concurrency control mechanism and a flexible trust model. As in YODA, ACE executes contracts asynchronously off-chain, decoupled from the consensus process. And similar to Arbitrum, execution is performed by a set of service providers that are appointed by the contract's issuer.

Such a model allows execution of complex contracts without slowing down the consensus process and enables flexible trust assumptions and liveness guarantees. Contract issuers can choose an appropriate set of service providers for each contract separately and users are free to choose which service providers they trust for contract safety and liveness. This execution model allows the use of a digital currency provided by a permissionless blockchain, with its transparency and integrity guarantees, while benefiting from higher efficiency and more flexibility from the off-chain execution.

ACE also has noteworthy differences to previous systems. Unlike Arbitrum, where service providers must reach unanimous agreement on the execution results (or the system falls back to an expensive on-chain verification), we enable more flexible verification, where execution results are accepted if at least $q$ out of $n$ service providers report the same result. That is, ACE supports a trust model where safety is ensured as long as fewer than $q$ of the appointed service providers are Byzantine faulty. In contrast to Ekiden, ACE does not require a TEE that is fully trusted for integrity (compromise of SGX platforms has been shown to be a relevant threat [6, 7]). And finally, unlike previous solutions, ACE supports cross-partition transactions (i.e. transactions that involve contracts handled by different sets of service providers), and thus enables safe and efficient execution of contracts that interact across service provider boundaries. The integrity of such cross-partition transactions is guaranteed even if faulty partitions are involved.
Concurrency control has been studied extensively in the context of database systems. Classical solutions include two-phase locking [12] and optimistic concurrency control [20]. Recent research has proposed deterministic alternatives [30], where transactions are pre-ordered before execution. The main benefit of such systems is that they avoid the need for expensive distributed commit protocols that require multiple rounds of communication between the involved partitions. Our observation is that the deterministic approach provides a good basis for off-chain smart contract execution and we tailor this approach for our purpose.

In particular, we augment the typical block structure with separate ordering and result sections. When a miner creates a new block, it pre-orders transactions for execution. Service providers examine new blocks and if the ordering section contains transactions with calls to their contracts, they execute them off-chain. Typically, concurrency control solutions require expensive synchronization between partitions, like distributed commit protocols. We avoid this cost by leveraging the pre-assigned order and broadcasting not yet committed transaction execution results to the peer-to-peer network. Once a transaction is fully executed, service providers sign a state-change transaction. Miners accept state changes signed by at least $q$ service providers and include it in the result section of one of the next blocks which commits the transaction.

ACE speeds up transaction processing by allowing service providers to use tentative contract states (previous execution results) that are not yet part of the chain. To ensure that no contradicting results that may obtain $q$ signatures are committed, each execution result includes a reference to the previous contract state and miners only include results that refer the latest on-chain state. This ensures that only the correct result is included and allows flexible trust models where $q$ can safely be less than $n / 2$.

Main results. The primary goal of ACE is to enable more complex contracts. To evaluate this aspect, we simulated the asynchronous off-chain execution of ACE and the sequential execution model (used in Ethereum). Our results show that ACE enables contract calls that take several orders of magnitude longer to execute (e.g., minutes instead of milliseconds) with similar throughput in terms of transactions per second. Thus, ACE significantly improves the main advantage of smart contracts, the ability to implement arbitrary financial applications based on cryptocurrencies.

Other advantages of smart contracts are improved transparency and liveness. ACE preserves the transparency, since contract execution results are publicly recorded on the chain. Naturally, ACE cannot provide the same liveness guarantees as Ethereum, since its contracts are executed by a set of pre-named entities instead of a permissionless network which makes Denial-of-Service attacks on specific contracts easier. However, ACE still provides strong and adjustable liveness guarantees, where contract calls are guaranteed to complete, as long as at least $q$ honest service providers are available.

Contributions. To summarize, this paper makes the following contributions:

- ACE system. We propose a novel system called ACE for offchain execution of complex smart contracts on permissionless blockchains. The key features of ACE are its flexible trust model and safe concurrency control protocol. 
- Evaluation. We show that ACE enables several orders of magnitude more complex contract calls compared to Ethereum.

\section{PROBLEM STATEMENT}

In this section, we motivate our work, define our main goal and discuss limitations of previous solutions.

\subsection{Motivation and Main Goal}

In Ethereum, all contracts are executed sequentially by all miners that participate in the consensus process. Such a sequential and globally-replicated execution model, combined with the PoW-based consensus mechanism, provides execution integrity and strict serializability [24] for transactions. Such a model also ensures the integrity of the blockchain's state (that includes the state of all contracts), assuming that the trust assumptions of Ethereum's consensus mechanism hold. For further background on Ethereum's execution and trust model, see Appendix A.1.

Ethereum can ensure transaction execution integrity only when all (honest) miners re-execute each transaction to verify the correctness of the result. This has two main problems: It represents a high cost for the miners in terms of verification time ${ }^{1}$ and it inherently limits the achievable throughput of the system and the types of contracts that can be implemented. To provide good throughput, the block interval must be kept small, and therefore the execution time of Ethereum contracts is limited. Such time limits have a direct effect on the allowed computational complexity of contracts.

Currently, the gas limit for each Ethereum block is approximately $8 \mathrm{M}$ gas. To put this into perspective, even the simple task of sorting 256 integers using insertion sort requires twice as much. Thus, although Ethereum is based on a Turing-complete language that in principle allows the execution of arbitrary smart contracts, the "arbitrary" part is severely limited in practice. For example, contracts that perform heavy cryptographic operations (e.g., for data feeds like TLS-N [28]) or use machine learning models are infeasible.

Our main goal in this paper is to provide a solution that allows safe execution of more computationally complex smart contracts (e.g. seconds instead of milliseconds of computation time) in permissionless blockchain systems like Ethereum. We argue that this would enable various new types of contracts, such as ones that perform cryptographic operations that are not natively supported by EVM. We assume that heavier computations that take hours or days to complete, are rarely needed in smart contracts.

\subsection{Limitations of Previous Solutions}

Recent research has explored alternative ways to execute smart contracts. In this section we summarize the main limitations of these approaches. Section 7 discusses additional related work.

Alternative execution models. In YODA [10] a subset of consensus participants is randomly sampled to execute and thus verify each contract call. The main limitation of systems like YODA is that each smart contract call needs multiple rounds of execution by separate subsets and the sampled subsets need to be relatively large

\footnotetext{
${ }^{1}$ Miners could spend the same time mining a new block and acquire monetary benefits The only direct benefit verification is "the common good" of the cryptosystem itself, and therefore miners are often tempted to skip the execution and verification, accepting the result as given, which is often referred to as "Verifier's Dilemma" [21].
}

(e.g., hundreds of nodes) to reduce the probability of cheating. Such approaches can reduce the required degree of execution replication compared to standard Ethereum (e.g., from thousands of nodes to hundreds), but they still require a great amount of redundancy, a multi-round execution process, large communication overhead, and the probability of integrity violation is not negligible. In addition, systems like YODA require an unbiased and distributed random beacon. State-of-the-art beacon protocols, like RandHound [29], require highly expensive initialization routines (that need to be repeated when new participants join or leave) and significant communication for every periodic random value.

In Arbitrum [17] the execution of smart contracts is performed asynchronously off-chain by a set of managers that are appointed by the contract creator. Execution results are accepted by miners, if all appointed managers signed the same results. If fewer managers sign results, the transaction does not immediately get accepted and a challenge period is entered instead. The signing managers and the disputing challenger are required to post a deposit and then one of them proves correctness of his results using a protocol that is logarithmic in the length of the execution trace. During the challenge period the contract cannot make progress. Cross-partition calls are handled by storing messages on chain that are equivalent to a new transaction, i.e., call-chains are not executed atomically, serializability is not guaranteed, and a long call-chain creates a high latency.

Ekiden [8] provides confidentiality for smart contracts and decouples contract execution from the consensus process by executing contract off-chain in SGX enclaves. While this approach also enables execution of more complex contracts, Ekiden requires that all enclaves are trusted, since compromising one enclave enables the adversary violate contract integrity arbitrarily. Recent research has shown that TEE compromise is a practical threat [5, 6, 13, 15, 22, 33].

Using distributed database terminology, each of the above solutions executes contracts in separate partitions. However, none of the solutions supports safe cross-partition transactions, i.e., contract calls from one execution partition to another. Since many Ethereum contracts make calls that write to another contract, these solutions therefore cannot execute them safely.

Sharding schemes. Sharding is a common approach to increase blockchain transaction throughput. One example scheme is called Chainspace [2] where the backbone infrastructure is divided into shards (partitions) and every smart contract author can designate a trusted shard to execute its smart contract, thus parallelizing execution. However, to maintain integrity across cross-shard executions all shards need to be honest and have mutual trust.

Omniledger [19] is another sharding scheme that supports crossshard transactions. Shards are selected using random sampling, similar to YODA [10]. This method requires large shard sizes (in the order of hundreds to thousands) to reduce the probability of cheating given this random selection. Thus, systems like Omniledger still have significant redundancy. In addition, Omniledger is designed for Bitcoin-style transactions and requires modifications to securely work for a smart contract system.

Transaction splitting. Another method to execute computationally complex contracts on Ethereum is to split the execution up into multiple transactions. However, splitting up contracts requires additional mechanisms to keep contract calls atomic, such as adding 
locks and timeouts within the contract (and is prone to subtle programming errors). Most importantly, splitting up contracts only works for a few individual cases, not for widespread use of computationally complex contracts. This approach cannot increase the average execution time per contract, since such contract calls are still limited by sequential execution.

\section{ACE OVERVIEW}

In this section, we provide an overview of our solution that is called ACE. The main goal of ACE is to enable safe execution of complex smart contracts in permissionless blockchains and to address the limitations of the previous solutions reviewed above.

\subsection{System Model}

Figure 1 shows our system model. ACE is based on a permissionless setting where any entity can freely take one of the following roles.

Clients are equivalent to users in systems like Ethereum. Clients can call smart contracts by broadcasting a signed transaction that specifies the called contract. In addition to calling contracts, clients can also deploy new smart contracts as contract creators by broadcasting a transaction that contains the contract code and its specification. The specification defines the service providers who should execute contract calls and acceptance criteria for execution results.

Miners are responsible for collecting and ordering transactions in blocks, similar to systems like Ethereum. ${ }^{2}$ The primary difference between ACE miners and Ethereum miners is that the former do not execute contract calls. Instead, they only perform basic validity checks for transactions and simple state-dependency checks for execution results before adding them to new blocks (see Section 3.5).

Service providers are responsible for executing contract calls. After execution is complete, service providers communicate the execution results through the network. Service providers must make their public key available to potential clients before they can execute smart contracts (e.g. by publishing it on a website or on the blockchain itself). Service providers can be incentivized through fees based on the execution complexity (see Section 7).

Network. We assume that the above entities communicate over a peer-to-peer network similar to existing blockchains such as Bitcoin and Ethereum.

\subsection{Execution Model}

The starting point of our solution is an off-chain contract execution model similar to Arbitrum [17]. At the time of deploying a new contract, the contract creator appoints $n$ service providers responsible for contract execution, called the executing set. The contract creator specifies the identities (public keys) of these service providers in a transaction that creates the contract.

Instead of requiring that all service providers have to agree on the execution results, we follow a different approach and allow the contract creator to define a quorum of $q$ service providers that are required for acceptable execution result. The identities of the

\footnotetext{
${ }^{2}$ We focus on Ethereum that uses Proof-of-Work consensus. Therefore, we use the term miner to refer to a consensus participant, although our solution is orthogonal to the used consensus scheme. ACE could be used also with other permissionless consensus schemes like Proof of Stake.
}

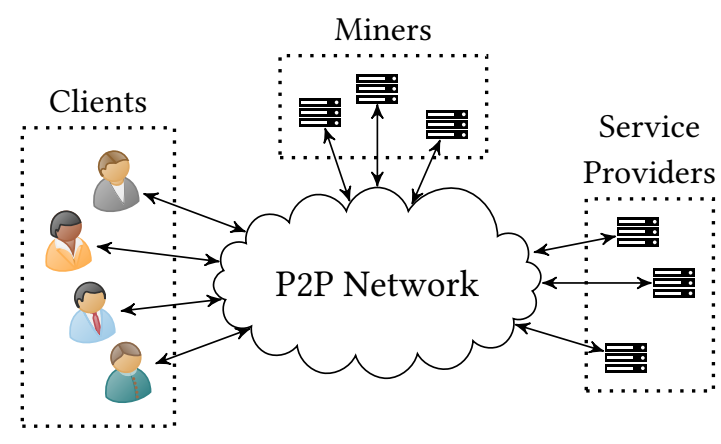

Figure 1: ACE system model. Clients deploy and call contracts by broadcasting transactions. Miners organize transactions into blocks. Service Providers execute contracts and post results to miners that will add them to new blocks.

$n$ service providers and the security parameter $q$ together define the trust level of the contract.

When a contract call is executed, if at least $q$ out of the specified $n$ service providers report the same execution result, the state change will be accepted by the miners. Contract calls involving multiple contracts need to be executed by the executing sets of all involved contracts, but do not require contract participants to trust executing sets of other contracts (see Section 3.4 for details).

Comparison to Ethereum. It may seem counterintuitive to allow the contract creator to choose the trust level for each contract separately. However, we emphasize that this design decision is in line with the existing trust model of Ethereum. For contract execution, Ethereum's trust model is contract-specific, i.e., users can freely choose which contracts they decide to trust. For example, if a user decides to send money to a particular smart contract, then with this action the user implicitly agrees with the contract specification, i.e., the conditions and the logic specified in that contract's code. A user that decides to trust one contract does not have to trust other contracts that run in the same system.

In ACE, this specification additionally includes the identities of $n$ service providers and the quorum size $q$. Similar to Ethereum, any party that does not use a particular ACE contract is not affected by any results of that contract.

More generally, our execution model can be seen as a hybrid of permissionless and permissioned systems. Both models have shown wide acceptance and ACE explores the space in between them (permissionless cryptocurrency, permissioned contracts). We argue that it is valuable to understand what kind of improvements that are possible with moderate trust model changes.

In particular, the decoupled off-chain execution provides more efficiency and allows for a flexible trust model in which a permissioned set of validators is appointed for each contract separately. The smart contracts that are executed off-chain can directly utilize the underlying digital currency that is implemented by the permissionless blockchain which provides transparency and doublespending protection. The trust model is flexible as it allows various applications with separate security requirements to co-exist in the same system. For example, even if all validators of one contract are fully compromised, only the funds of that contract are affected and the integrity of the currency and other contracts are still guaranteed 
for all other parties. Additionally, using a public blockchain to connect clients to service providers enables simple service discovery.

\subsection{Trust Assumptions}

We consider clients fully untrusted, similar to most permissionless blockchain systems. Regarding miners, we follow the standard trust assumptions of Ethereum and other PoW systems. Any individual miner can be malicious, but miners are collectively trusted for consensus (honest majority of the mining power).

Service providers can be reputable entities such as well-known companies who run contracts in exchange for a small service fee or non-profit organizations who run contracts for public good. We assume that some service providers may get compromised and behave arbitrarily (in contrast to systems like Ekiden). Each contract creator appoints an executing set and defines the security parameter $q$ for the deployed contract. We say that a contract has an honest quorum, when there are $\geq q$ non-faulty service providers in the set. With $\geq q$ Byzantine faults, we say that the contract has a faulty quorum. Note that a contract may have both an honest and a faulty quorum, since $q$ can be $<n / 2$. The integrity of the underlying cryptocurrency (e.g. money creation, double-spending) is independent of the contract execution and does not rely on any of the appointed service providers.

\subsection{Requirements}

Next, we define informal requirements for our solution. Section 5 provides more detailed definitions and proofs.

Requirement 1: Safety. The main safety requirement of ACE is that it should enable safe cross-partition calls without having to assume all partitions trusted. More precisely, our solution should guarantee execution integrity and strict serializability for all the transactions of a contract that does not have a faulty quorum (i.e., it has $<q$ Byzantine faulty service providers) even if the contract interacts with other contracts that have faulty quorums ( $\geq q$ faults) This requirement sets ACE apart from previous solutions as none of them supports such safe cross-partition calls.

Strict serializability [24] is a common isolation property for distributed database systems. It ensures that the outcome of transactions executed in parallel (in separate partitions) is equal to one in which the transactions had been executed atomically and sequentially in the order apparent to an external observer. For further background on concurrency control, see Appendix A.2.

Execution integrity, in our context, means that the code of the contract is executed correctly given all inputs that the contract receives. If the execution of one contract depends on an input from another contract, two options are possible. The first option is that the contract's logic needs to treat such inputs as untrusted data and use them accordingly. The second option is to consider such inputs as trusted data which means that clients should use the contract only if they are willing to trust the source contract (and its trust level) as well. In both options, a faulty source cannot violate the integrity of the main contract's execution.

Note that this is similar to contracts in Ethereum today. If an Ethereum contract receives input from, e.g., an oracle or another contract, this input either needs to be treated as untrusted by the main contract's code or alternatively the oracle or the input source contract code needs to be considered trusted by the users of the main contract.

Requirement 2: Liveness. For liveness, we consider two properties. The first is called contract liveness which means that any contract with an honest quorum ( $\geq q$ non-faulty service providers) stays responsive, even if it interacts with other contracts that do not have honest quorums or have faulty quorums. When we say that a contract is "responsive", we mean that it is able to complete all transactions either by successfully executing them or by returning an abort message, and then move on to processing the next transaction. The main benefit of such a liveness property is that interaction with another potentially faulty or non-responsive contract cannot halt the main contract.

The second liveness property that we consider is transaction liveness which means that a transaction will eventually be executed successfully. In Section 5.2 we show that ACE provides transaction liveness when each contract involved in a transaction has an honest quorum and none of them has a faulty quorum.

Requirement 3: Performance. The main performance requirement of ACE is efficient concurrent control. In distributed systems, the entities that execute transaction need to agree on the order of execution and whether execution was successfully completed on all nodes before committing the final results. Classical distributed commit protocols like two-phase locking [12] guarantee strict serializability, but require multiple rounds of communication between all involved entities (see Appendix A.2).

ACE should minimize such concurrency control overhead for transactions that are executed within one contract and across multiple contracts. In particular, we want to optimize the common and benign case, so that safe transaction execution has minimal communication overhead in the absence of errors and attacks (while recovery from error conditions can be more expensive).

\subsection{Contract Execution Overview}

Next, we provide an overview of contract execution in ACE. Similar to Ethereum, clients broadcast transactions to the peer-to-peer network. We assume that every ACE transaction contains at least one write operation, as read-only computations are possible simply by examining the blockchain without issuing a new transaction. The broadcasted transactions are ordered and collected into blocks by miners. If a transaction does not include any contract call (i.e., it only transfers money from one account to another), the miners immediately execute the transaction on-chain and record it in the result section of the block.

If a transaction calls a contract, the miners perform basic checks (e.g., correct transaction format) and then include the transaction in the ordering section of a block, but importantly do not execute the contract call. The new block is broadcast to the network. Once the service providers appointed for this contract receive it, they execute the contract call code. After executing the call, service providers sign so called state change transactions and broadcast them back to the network. Once the miners receive signatures from a quorum $q$ of the appointed service providers on the same set of state changes, they check state-dependency, meaning that the state changes refer to the hash of the previous state, and then include the state changes and the corresponding signatures in the result section of a future 
(not necessarily the next) block. The state-dependency check ensures that no conflicting results from two separate quorums within the same execution set can be included and that results cannot be replayed in different contexts (e.g. if another fork of the blockchain becomes the canonical chain).

Safe and efficient commits. In distributed systems, nodes need to agree whether execution was successfully completed in the same order on all nodes before committing the results. In ACE, the service providers do not run an agreement protocol, because transaction ordering is already performed by the miners. Similarly, the miners ultimately decide whether a transaction is committed (by including it to the next block), and therefore the service providers not do have to run a multi-round commit protocol with Byzantine agreement. Thus, ACE can be seen as a variant of deterministic concurrency control [27], where transactions are pre-ordered to avoid expensive distributed commit protocols (see Appendix A.2).

In distributed databases, transactions are typically committed immediately after their execution to avoid so called dirty reads where one transaction changes the state of a resource and another transaction reads the changed state before the first transaction is committed. If the first transaction cannot commit (e.g., due to a crash), the second transaction must be rolled back to preserve consistency.

In off-chain contract execution transaction commits have inherent latency, due to the infrequent block generation rate of permissionless blockchains. Thus, dirty reads are in practice unavoidable. ${ }^{3}$ ACE ensures consistency in the presence of potential dirty reads as follows. When miners include signed state changes in a new block (i.e., commit transactions), they need to ensure that the state changes are added per contract in the same order as they were listed in the ordering section previously. That is, a transaction that appears before another transaction in the ordering section needs to either have its state changes committed or be invalidated (see Section 4.6) before state changes of the second transaction can be committed to a block if the two transactions affect the same contract state.

\subsection{Replicated Cross-Contract Execution}

A common way to execute cross-partition transactions in distributed systems is that nodes (service providers) execute those operations that access their resources (contract storage variables) and they would send a request to another partition when the executed transaction reads or write to a resource that is controlled by the other partition. The contacted service provider would then execute the requested operations and send back a result. This common approach that optimizes for computational resources is illustrated in Figure 2 on the left. However, a significant drawback of this approach is that it may require lots of communication. A single smart contract call may involve several subcalls to many other contracts which would in turn mean many rounds of communication between the involved service providers. This can have an especially drastic effect in an adversarial setting where the partitions do not trust each other, as in our case.

Because of this, we follow a different approach in ACE and optimize for communication cost. Instead of forcing the service

\footnotetext{
${ }^{3}$ In principle, it would be possible to design a system, where each executed transaction is committed to a new block before the execution of the next transaction is allowed to proceed. However, such system could process, in the worst case, only one transaction per block, and be completely impractical.
}

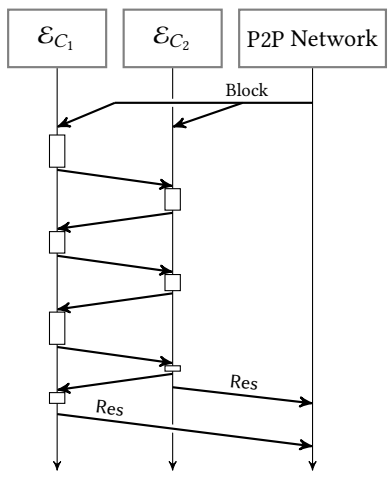

(a) Strawman solution

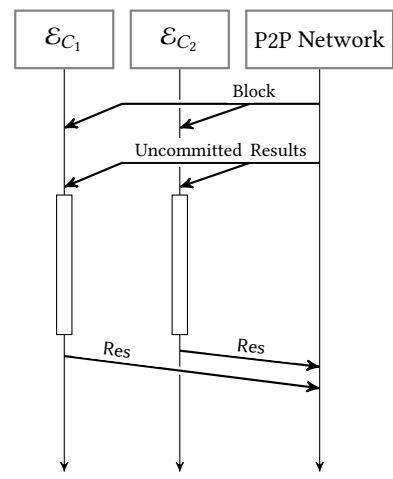

(b) Contract execution in ACE
Figure 2: On the left we have the strawman solution for concurrent execution where each subcall requires communication between partitions. On the right we have our replicated model where all involved service providers execute the complete transaction preceded by an initial update of the other contracts tentative state based on uncommitted results.

providers to communicate before and after every subcalls, all involved service providers execute the full transaction and publish their results afterward, as illustrated on the right in Figure 2. To enable such cross-partition calls, the involved service providers only need to update tentative states of involved contracts before executing the call. This is necessary since some results of previous contract calls may not have been included in the blockchain yet.

To do this, service providers listen to state change results that are broadcast to the P2P network. If the required state updates (results for relevant preceding transactions) are not received within a specified timeout, the service provider can issue an abort for the transaction (see Section 4.6). Otherwise, the service provider can start executing the transaction. We use the P2P network for exchanging the tentative contract states, because it makes deployment of ACE easier. In particular, the service providers do not need to establish direct communication links between each other which gives them the freedom to migrate, change IP addresses etc. ${ }^{4}$

Such cross-partition contract call model requires that every transaction has to list all possibly involved contracts. In ACE, clients pre-run transactions as a reconnaissance step to determine the set of contracts and service providers involved and attach this information to the broadcast transaction (see Section 4.3). This enables service providers to know which contract calls they need to execute and for which contracts they need to assemble the tentative state. Based on this tentative state, all of the involved service providers execute the full call chain.

After the execution has finished, each service provider signs the results (list of state changes) and sends them to the P2P network. Once miners have received the threshold number of signatures on the results for each of the involved smart contracts (and performed the required state-dependency checks), they will include them in the results section of a future block (again, following the per-contract order from the previous ordering sections).

\footnotetext{
${ }^{4}$ If some service providers communicate often, they can ensure that they are well connected within the P2P network for reduced latency, similar to what large mining pools do to ensure low latency.
} 


\section{ACE DETAILS}

In this section we describe the ACE system in detail.

\subsection{Block Structure}

ACE blocks consist of two sections: The Ordering section is used to list and order transactions before execution, and the Result section lists the state changes of executed smart contract calls.

\subsection{Contract Deployment}

Deployment of a new smart contract $C$ is shown in Figure 3 and works as follows. (1) The client chooses an executing set $\mathcal{E}_{C}$ out of the available service providers $\mathcal{P}$ and sends a deployment request $R_{D}$ to each service provider $P \in \mathcal{E}_{C}$ asking them to act as executors for this contract deployment. The request contains the contract code, parameters $n$ and $q \leq n$, and the identities of the chosen service providers. (2) Every service provider that agrees to be part of the executing set returns a signature $\sigma_{P}\left(R_{D}\right)$ on the deployment request. (3) The client creates a deployment transaction $\mathrm{Tx}_{D}$, containing the list of service providers from $\mathcal{E}_{C}$, the deployment request $R_{D}$, and the set of signatures $\Sigma_{\mathcal{E}_{C}}\left(R_{D}\right)=\left\{\sigma_{P}\left(R_{D}\right) \mid P \in \mathcal{E}_{C}\right\}$ from all members of $\mathcal{E}_{C}$. The client sends the deployment transaction to the network, where miners verify the signatures and, if valid, include it in the ordering section of a new block. (4) Once the transaction is included, the members of $\mathcal{E}_{C}$ execute the constructor of the contract like a normal contract call, as described below. (5) The service providers send the result Res to the network to be included in the result section of a future block.

\subsection{Contract Call}

To call a smart contract, a client creates a transaction similar to Ethereum transactions. However, in addition to the standard components of an Ethereum transaction (nonce, gas, gas price, recipient, transfer value, input data, signature) the client also specifies the set of involved contracts which allows service providers to quickly determine whether they need to be involved in the contract execution. While the directly called contract is always specified for Ethereum transactions as well, the same does not apply to contracts that are indirectly called as subcalls from the executed contract.

To determine the set of involved contracts, the client can first execute the contract locally to get a set of dynamically called contracts. Such a reconnaissance step is analogous to how gas costs are estimated in Ethereum. Similar to Ethereum, the contract state may change between local pre-execution that is based on the latest block and the final execution where new transactions may have been already applied. Such transaction execution may need to abort, and the client needs to send the transaction again with newly determined set of involved contracts (see Section 4.6). However, this is unlikely to happen for well-written smart contracts that are not purposefully designed to exhibit such behavior. Alternatively, static analysis can be used to over-approximate the involved contracts.

We note that clients cannot misuse this feature to violate contract safety. If the contract call has a subcall to some contract that is not included in the list, it will simply not be executed by the responsible executing set, i.e. no changes to the respective contract state can be committed. Other (non-faulty) executing sets involved in the transaction will simply produce an exception during the execution.

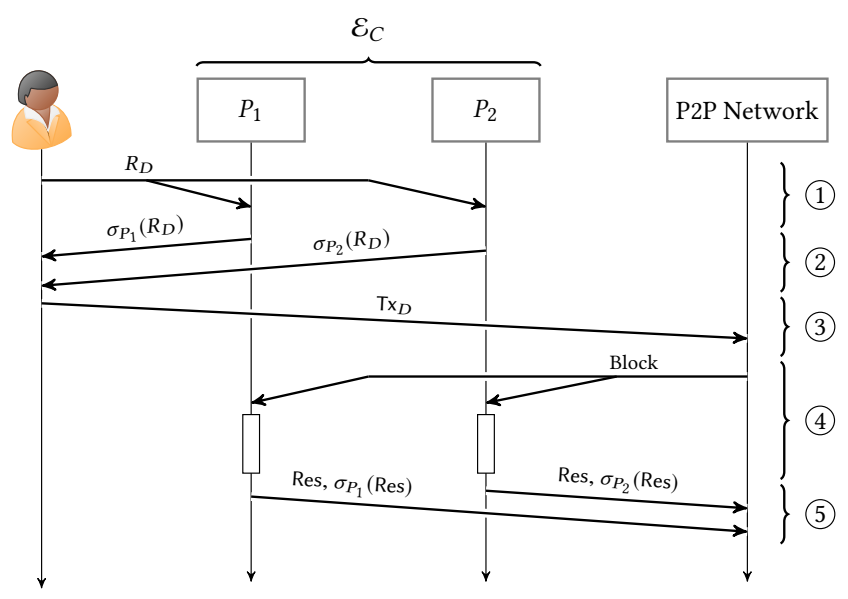

Figure 3: Contract deployment. The client first sends the deployment request $R_{D}$ to the service providers chosen to be in the executing set $\mathcal{E}_{C}(1)$, who then sign it and return it to the client (2). The client sends the resulting transaction to the network (3), where it gets included into a block. The service providers see this transaction in a block, execute the contract constructor (4) and send the signed results to the network to be included in a future block (5).

Also note that a per-transaction gas limit (and a gas price, see Section 7) is still specified by the client to prevent infinite loops, even though there is no block gas limit in ACE (in contrast to Ethereum).

Once the client has sent the transaction to the network, the miners check its validity, i.e., they check whether the sending account has enough balance, the transaction nonce is correct, and the signature verifies correctly. They then include the transaction in the ordering section of the next block.

Service providers parse the blocks they receive and check whether they are involved in any contract call, i.e. whether some transaction has specified a contract for which they are responsible. If this is the case, they add the transaction to their execution queue. Service providers can parallelize execution of distinct smart contracts, i.e. they only need to execute calls to the same contract sequentially and can thus use separate queues for each contract.

To execute a transaction that does not involve other smart contracts, the service providers simply execute the transaction, sign the state changes and send them to the network. The miners then check that the state transition is based on the most recent state, i.e., that the previous state hash stored in the result is equal to the hash of the current state before applying the results. This ensures that results cannot be replayed in different contexts and no conflicting results can be included even if different results have a quorum of signatures. The miners then check that the transaction has been signed by a quorum of $q$ service providers in the executing set. If this is the case, they include the results in the result section of the next block, and thus commit the transaction.

\subsection{Cross-Partition Contract Call}

If the transaction does involve other smart contracts, the contract call is executed as described below. An example is shown in Figure 4. 
(1) Sending the Transaction. The client creates transaction Tx and sends it to the network as described in Section 4.3. Miners check its validity and include it in the ordering section of the next block.

(2) Fetching the Block. The service providers fetch the new block including the transaction Tx from the P2P network and, if the transaction is addressed to (in the example, contract $C_{1}$ for $P_{1}$ and $P_{2}$ ) or lists (in the example, contract $C_{2}$ for $P_{3}$ ) a contract they are responsible for, they add the transaction to the execution queue of the respective contract.

(3) Tentative State Update. The service providers continue to receive tentative results from other transactions from the $\mathrm{P} 2 \mathrm{P}$ network that have been signed by a quorum of the respective executing set but not yet been included in the chain. Based on these results, the service providers update the tentative local state for other contracts. This allows them to use the current state of other service providers involved in the contract call during execution. In Figure 4 $\mathcal{E}_{C_{1}}$, i.e. service providers $P_{1}$ and $P_{2}$, needs updates for the state of contract $C_{2}$, and $P_{3}$ needs updates for the state of $C_{1}$, if other transactions to one of these contracts is scheduled before $T x$.

(4) Contract Execution. Once the states have been updated and the transaction $\mathrm{Tx}$ is at the top of the execution queue for each contract, every service provider executes the full transaction, i.e., the contract call with all subcalls based on the current (tentative) state of the smart contracts.

(5) Broadcast Result. After the execution, each service provider signs the resulting state changes and sends the results (list of state changes) and their corresponding signature to the P2P network. The results also include a hash of the previous contract state to ensure that e.g. blockchain forks or inconsistent tentative states of other contracts cannot lead to inconsistencies. At least a quorum of $q_{i}$ service providers in each involved executing set $\mathcal{E}_{C_{i}}$ has to sign the state changes for the transaction to be accepted. In the example in Figure $4, P_{2}$ becomes unresponsive and does not send its results. The protocol can still progress without abort, since $P_{1}$ is sufficient to achieve a quorum in this case $(q=1, n=2)$.

Upon receiving state changes from service providers, miners check that previous state changes have already been received and applied, i.e., that for every involved smart contract, the state changes correspond to the next scheduled transaction in the list of transactions given by the ordering sections of the blocks in the chain. They further check the signatures on the state changes, ensuring that a quorum $q_{i}$ has been reached for all involved smart contracts and then include the state changes in the result section of a future block.

\subsection{Block Verification}

To verify a block, verifiers need to check that it was formed correctly, the transactions listed in the ordering section are valid transactions, and that the results are valid. Further, they have to check that the block conforms to consensus rules, e.g. that it has a valid proof-ofwork, and finally apply the state changes listed in the results section.

To check the validity of the ordering sections, the node checks for every transaction that the signature from the sender is valid and that the sender has sufficient balance for the call. For each result in the result section, the node checks that the signatures from the service providers are valid and that a quorum from each executing set of involved contracts has been reached and it performs a

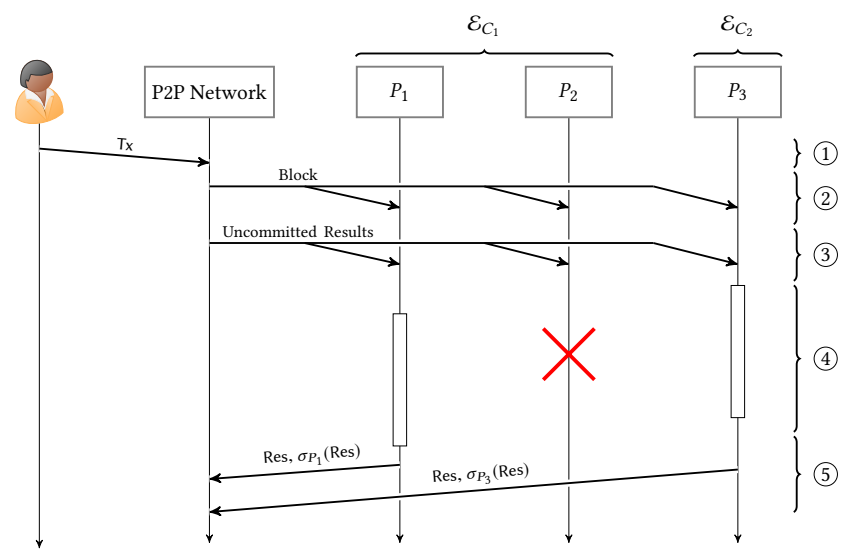

Figure 4: Cross-partition contract call. In this example, contract $C_{1}$ is executed with a 1-out-of-2 quorum by $\mathcal{E}_{C_{1}}=\left\{P_{1}, P_{2}\right\}$ and $C_{2}$ is executed with a 1-out-of-1 quorum by $\mathcal{E}_{C_{2}}=\left\{P_{3}\right\}$. The diagram shows the messages exchanged in a contract call involving $C_{1}$ and $C_{2}$ in which $P_{2}$ becomes unresponsive after the state exchange. Since $P_{1}$ is responsive, a quorum can still be reached and the results can be committed successfully.

state-dependency check, i.e. it checks that the hash of the previous state stored in the result is equal to the current state (before applying the state changes) of the contract. Additionally, it checks that all results of previous transactions involving one of the involved smart contracts have been applied. That is, the previously defined per-contract transaction order is preserved. Nodes can keep FIFO queues for transactions per contract to easily keep track of which results are expected next for each smart contract.

\subsection{Recovery from Errors}

Unresponsive service providers. If $u$ individual service providers from one set become unresponsive, the transactions handled by that set of service providers can still complete, as long as $u \leq n-q$. Section 4.4 describes an example, where transactions complete despite one unresponsive service provider.

Unresponsive executing set. If $u>n-q$ service providers from the same set become unresponsive, we say that this set is unresponsive, and transactions involving that set of service providers can obviously not be processed, until some of the unresponsive service providers are fixed, e.g., restarted after a crash (in Section 7 we discuss how permanently unresponsive service providers can be replaced). The goal of ACE is to ensure that in such cases the unresponsive set cannot prevent other contracts from proceeding.

ACE achieves this by allowing service providers to sign and broadcast an abort for a transaction. The service providers will broadcast an abort if they do not receive tentative state updates (with $q$ signatures) for involved contracts in cross-partition transaction execution within a pre-defined time limit. They can also broadcast an abort if they do not receive enough signatures on the new state after execution within some time limit after they have finished its execution. 
If the miners receive a signed abort from at least $q_{i}$ service providers out of an executing set $\mathcal{E}_{C_{i}}$ of one of the involved contracts $C_{i}$, where $q_{i}$ is the quorum threshold of $C_{i}$, they can include these aborts in the blockchain as result for the given transaction instead of state changes. This allows involved contracts to progress with the execution of future transactions, if the executing set of another contract is unresponsive for an extended period of time.

Failed reconnaissance. If a client failed to correctly determine the set of contracts involved in a transaction, the service providers will notice this at the time of transaction execution and produce an exception as execution result (similar to existing Ethereum exceptions). The client can issue the same transaction again with newly estimated involved contracts.

Missing state change. Contract calls in ACE may involve dirty reads. If the state change from the previous transaction never reaches miners (e.g., due to a temporary networking issue or intentional attack), the following state changes cannot be committed, as the miners must follow the per-contract order for transactions. In such cases, the service providers will soon observe that their state changes are not committed by miners and they can simply re-send the state changes again.

\section{SECURITY ANALYSIS}

In this section we analyze the safety and liveness of ACE.

\subsection{Safety}

Recall from Section 3.4 that the main safety requirement of ACE is to ensure that contracts without faulty quorums provide execution integrity and strict serializability even if they interact with faulty contracts. We perform this analysis in two steps.

Intra-partition safety. We start our analysis by showing safety for contract calls involving a single smart contract.

Claim 1. Given the specification of a contract, which defines an executing set $\mathcal{E}$ consisting of $n$ service providers and quorum $q$, the following holds in asynchronous networks: If fewer than $q$ service providers from $\mathcal{E}$ have Byzantine faults, execution integrity and strict serializability hold for this contract for contract calls involving no other contracts.

Proof. Since less than $q$ of the members of $\mathcal{E}$ are compromised and all others correctly execute the contract code, the adversary cannot produce $q$ or more signatures on state changes that are different from the correct execution. Since all non-compromised service providers follow the transaction order per contract, and execute their transactions atomically and sequentially one after another, and since miners check state-dependency of results, execution integrity and strict serializability is provided for all such transactions.

Cross-partition safety. Next, we show that safety holds for contract calls involving multiple smart contracts.

CLAIM 2. Given the specification of contract $A$, which defines an executing set $\mathcal{E}_{A}$ that consists of $n_{A}$ service providers and quorum $q_{A}$, the following holds in asynchronous networks: If fewer than $q_{A}$ service providers from $\mathcal{E}_{A}$ have Byzantine faults, execution integrity and strict serializability hold for contract $A$ even in contract calls involving other contracts.

Proof. We distinguish three possible cases:
1. No faulty executing sets. If for every other involved contract $B$ with $\mathcal{E}_{B}$ consisting of $n_{B}$ service providers and quorum $q_{B}$, less than $q_{B}$ service providers from $\mathcal{E}_{B}$ are Byzantine faulty, the adversary cannot produce an acceptable and false state change for contract $B$ and thus the adversary cannot produce a false tentative state of contract $B$ that would be accepted by any of the non-faulty service providers of $\mathcal{E}_{A}$. Because of this, any results signed by $q_{A}$ or more providers from $\mathcal{E}_{A}$ are derived from the correct execution of the contract based on correct initial states of all involved contracts. Thus, execution integrity is guaranteed for all executed transactions.

2. Faults during execution. If for all involved contracts, the tentative states are correct, but for at least one involved contract $B$ with $\mathcal{E}_{B}$ consisting of $n_{B}$ service providers and quorum $q_{B}$, at least $q_{B}$ service providers from $\mathcal{E}_{B}$ become Byzantine faulty during the execution, they can produce a false result for the execution of the contract with a quorum from $\mathcal{E}_{B}$. However, due to the same reasons as in Claim 1, no quorum from $\mathcal{E}_{A}$ can be achieved on these results, which ensures that they will not be accepted by the miners and thus execution integrity is ensured.

3. Inconsistent tentative state. If for an (or all other) involved contract $B$ with $\mathcal{E}_{B}$ consisting of $n_{B}$ service providers and quorum $q_{B}$, at least $q_{B}$ service providers from $\mathcal{E}_{B}$ are faulty before the execution, they can produce inconsistent results for the execution of previous calls to $B$, i.e. they can convince members of $\mathcal{E}_{A}$ to use a false tentative state of contract $B$ for the contract execution. Once the contract has finished executing, a quorum of $\mathcal{E}_{A}$ may sign a set of state changes based on this false state. However, the signed results include a reference to the tentative state, and miners only include state changes in the result section of a block if this correctly references the previous state committed to the chain, i.e. the results will only be included if the tentative state used for the execution is in fact the canonical state committed to the chain, thus ensuring execution integrity for contract $A$.

In all three cases, strict serializability is ensured due to the total order given by the ordering sections of the blockchain and because all dependent transactions are executed atomically based on this order. This guarantees that all concurrent executions are equivalent to the sequential execution according to this order.

Finally, we note that the execution results (state changes) of smart contracts are only relevant to participants of the smart contract, as long as they do not enable double spends or affect the overall supply of the cryptocurrency. In ACE, the integrity of the underlying cryptocurrency is guaranteed independently of smart contract execution. In particular, a smart contract (or a faulty executing set) is not able to arbitrarily modify its balance. Transfers of money is always checked on the cryptocurrency consensus layer, i.e. a contract balance can only increase by amounts explicitly sent in transactions (or received from other smart contracts) and smart contracts can only initiate sending of funds that do not exceed their balance. This ensures that compromised executing sets can neither double spend funds nor create money out of nothing, even if an executing set is completely compromised.

\subsection{Liveness}

Recall that we consider two liveness properties. Contract liveness means that a contract with an honest quorum is responsive even 
if it interacts with other non-responsive or faulty contracts. Transaction liveness means that a transaction is eventually executed successfully, when each involved contract has an honest quorum and none of them has a faulty quorum. Next, we show how ACE provides these properties.

We note that the previously discussed safety properties hold in asynchronous networks, but for liveness we need to assume a synchronous network (i.e., known bound on network delays). Synchrony is a common assumption in many consensus protocols, including Bitcoin [25].

Contract liveness. We make the following claim for contract liveness:

CLAIM 3. Given the specification of contract $A$, which defines an executing set $\mathcal{E}_{A}$ that consists of $n_{A}$ service providers and quorum $q_{A}$, the following holds in synchronous networks: If at least $q_{A}$ service providers from $\mathcal{E}_{A}$ are non-faulty, then all transactions to contract $A$ are eventually completed (i.e., executed or aborted).

Proof. There are two possible cases to consider. First, we consider the case where contract $A$ does not call any other contract. Given that at least $q_{A}$ non-faulty service providers exist, the execution is completed successfully for calls to contract $A$, since the non-faulty service providers sign execution results.

Second, we consider the case where contract $A$ interacts with another contract $B$. If the non-faulty service providers of $\mathcal{E}_{A}$ receive state changes from $\mathcal{E}_{B}$, they can execute the call successfully and sign execution results. If the available service providers of $\mathcal{E}_{A}$ do not receive state changes from $\mathcal{E}_{B}$ after a timeout, they issue an abort which allows other transactions for the same contract to be completed (executed or aborted). Similarly, if the available service providers of $\mathcal{E}_{A}$ receive inconsistent state changes from $\mathcal{E}_{B}$ (because contract $B$ is faulty), the transaction will be aborted which allows other transactions for contract $A$ to be completed.

Transaction liveness. We make the following claim for transaction liveness:

Claim 4. Given a transaction Tx recorded in the ordering section of a block and involving $k$ contracts $C_{i}(1 \leq i \leq k)$ that define executing sets $\mathcal{E}_{C_{i}}$ with quorum $q_{i}$, the following holds under synchronous network assumptions: If $b_{i} \leq \min \left(q_{i}-1, n_{i}-q_{i}\right)$ service providers from $\mathcal{E}_{C_{i}}(1 \leq i \leq k)$ have Byzantine faults, liveness is guaranteed for transaction Tx, i.e. Tx will be eventually executed.

Proof. Since only $b_{i} \leq \min \left(q_{i}-1, n_{i}-q_{i}\right) \leq n_{i}-q_{i}$ of the service providers of $\mathcal{E}_{C_{i}}$ are faulty, each involved contract has a quorum of non-faulty service providers and each of the involved contracts provides liveness (Claim 3), i.e., they will broadcast results of all previous transactions within a finite bound (given the synchrony assumption) which allows the involved service providers to derive the tentative state for all involved contracts.

Following this, no honest service provider will issue an abort, and aborts issued by Byzantine service providers will not reach the quorum threshold (since $b_{i} \leq q_{i}-1$, i.e., no faulty quorums), allowing contract execution by all honest service providers. Since there exists an honest quorum for each involved contract, the execution results receive the necessary quorums and are again dissipated within a fixed time bound, after which no honest service provider issues an abort and aborts issued by Byzantine service providers will not reach the quorum threshold (since $b_{i} \leq q_{i}-1$ ), allowing the inclusion in the blockchain.

\section{EVALUATION}

In this section we analyze how complex contracts ACE can support and evaluate the performance of ACE.

\subsection{Contract Complexity}

We compare the average computational complexity of contract calls supported in the ACE execution model to the sequential execution model used in Ethereum. We modeled our system in Python to simulate its throughput for a large number of nodes. This allowed us to detemine the complexity of contracts supported given a target throughput and different dependencies between the contracts.

Simulation details. To simulate ACE, we first set parameters such as the block size, the total number of service providers, the total number of smart contracts, and the mean and standard deviation for contract execution time. The block size puts an upper bound on the throughput and the number of service providers and contracts affects how interdependent the smart contract executions are.

We generate a set of simulated contracts that are defined by their execution time and the number of involved contracts (number of sub-calls). For simplicity we assume that each contract call to the same contract results in the same amount of sub-calls and the execution time is fixed. The execution time is sampled from a normal distribution with given mean and standard deviation while the involved contracts are sampled uniformly from the list of all contracts. Furthermore, each contract is assigned a set of (between 1 and 6) responsible service providers, chosen uniformly from fix-sized list of providers.

For each block, we then sample the transactions for its ordering section. Each transaction calls a contract sampled uniformly at random from our set of generated contracts.

In our simulations, we simulated service providers that are well connected to each other with connection speeds of $1 \mathrm{Gbps}$ and $50 \mathrm{~ms}$ network delays to simulate propagation delay during the exchange of the tentative state.

Measurements. For each configuration, we measured the throughput in transactions per second (Tps) for ACE for different mean execution times of the contracts. This allows us to determine how computationally intensive smart contract executions can be, given a target throughput (i.e. we want to exhaust throughput of an underlying consensus layer that can achieve $k$ Tps). For our experiments, we fixed a block interval of 15 seconds and simulate up to 15000 transactions per block (1000 Tps). Note that this upper bound is not achievable in permissionless systems given current network throughputs, i.e. in practice the throughput will be capped at a lower number. Given the current average Ethereum block size, the throughput would be limited to $10 \mathrm{Tps}$. The total number of service providers in our experiments is set to 100 .

In order to compare to the theoretical maximum execution complexity in Ethereum and similar systems for a given target throughput, we also show the execution time supported by a sequential transaction execution model. This gives an absolute upper bound for the sequential execution model, since it assumes an instant propagation of the block and transactions and no other overheads, 


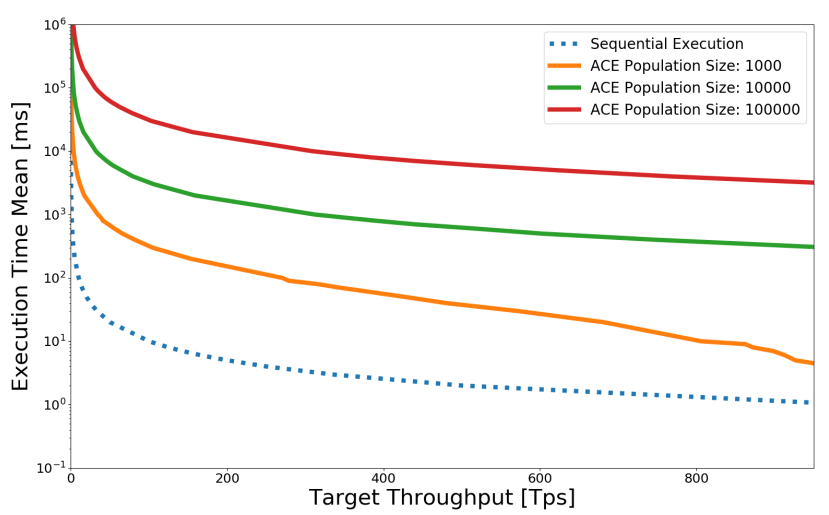

Figure 5: Average execution time per transaction given a target throughput. Sampling size refers to the number of different contract in the system from which we sample our transactions. The number of additionally involved contracts per contract call are sampled uniformly from the range $[0,5]$.

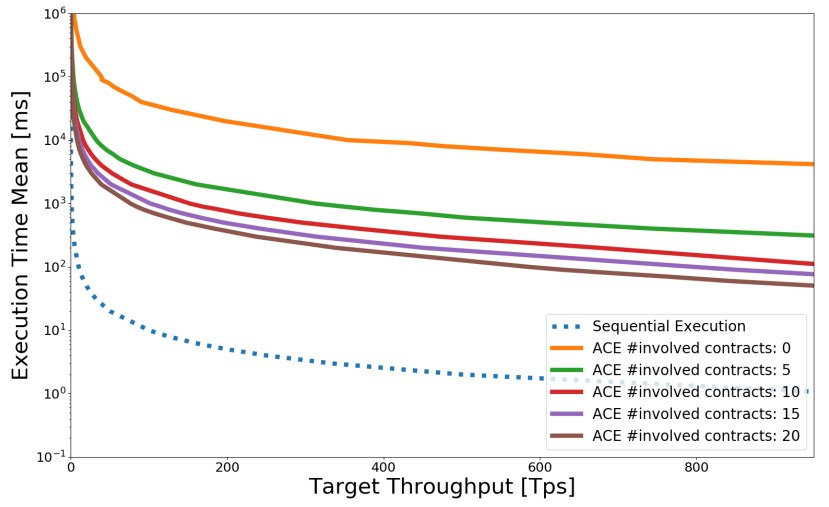

Figure 6: Average execution time per transaction given a target throughput. The sampling size is fixed to $10^{\prime} 000$ different contracts while the upper limit of the range from which we sample the number of additionally involved contracts varies from 0 to 20 .

whereas in reality the average execution time has to be much lower to achieve a given target throughput in a sequential execution model (such as Ethereum).

Results. Figure 5 shows the results for different numbers of contracts from which we sample transactions. The maximum number of involved contracts is fixed at 5 which is currently the 99th percentile in Ethereum's mainnet. The impact of the sampling size is clearly visible from our simulation results.

When sampling from a small set of 1000 different contracts, ACE allows supporting execution times of one to two orders of magnitude higher than sequential execution, because the dependency between transactions is high. For a set size of $100^{\prime} 000$ contracts, much higher execution times can be supported for a given target throughput. For example, for a target throughput of $10 \mathrm{Tps}$ (currently achievable by Ethereum), transactions can have a mean execution time of 5 minutes, whereas the theoretical upper bound for sequential execution is at 0.1 seconds.

In Figure 6, we show the results for varying numbers of other contracts involved in a contract call. For these measurements, contracts are sampled from a set of 10 ' 000 contracts. The numbers listed in the graph denote the upper bounds of the range from which we uniformly sample the number of additionally involved contracts for a contract call. We see that going from transactions involving zero additional contracts to transactions involving up to 5 additional contracts adds a lot of dependency and therefore supports a much lower average computational complexity. However, this difference gets much smaller with more and more additionally involved contracts.

Our results show that ACE is capable of handling contracts with execution times in the order of seconds or minutes. Clearly, ACE can support much longer execution times when contracts have a low interdependence, e.g. if many distinct (e.g. 10'000) smart contracts exist in the system or contracts only involve a low number of other smart contracts. Also in our simulations we used 100 service providers. If more service providers are available, ACE can support even longer execution times.

Summary. Overall, we observe that ACE can process transactions with execution times that are 2-4 orders of magnitudes larger than what can be theoretically handled by sequential execution in the optimal case (instant block propagation). Since sequential execution is much slower due to block propagation delays, the difference would be even larger in practice.

\subsection{ACE Performance}

Next, we evaluate the computation, storage and communication cost of ACE.

Implementation. We implemented ACE based on Ethereum. Our implementation supports the execution of smart contracts that are compiled to EVM bytecode and it is based on the EVM implementation from the Parity Ethereum client.

Computation cost. The first performance metric that we evaluate is computation, measured as the time required for verification of contract call results (state changes). This verification task needs to be performed by all miners (and all other nodes that verify blocks) and if verification takes too long, then the savings of off-chain contract execution may be reduced or eliminated.

Verifying the transaction result consists of the verification of the service provider signatures, deserialization of the encoded results and finally application of the state changes. As the time used for hashing (needed for signature verification) and deserialization both depend on the size of their input we measured the verification time relative to the size of a transaction result in terms of number of storage changes, i.e. the number of variables changed during the contract call, which is independent of the number of sub-calls or the quorum size. One variable corresponds to 64 Bytes (key and value).

We conducted our experiments on a machine with an Intel(R) Core(TM) i7-7700 CPU @ 3.60 GHz, 2 threads and 16 GB RAM. As expected, the verification time is proportional to the result size. The throughput for verifying and applying state changes is roughly 160 $\mathrm{MB} / \mathrm{s}$. Each service provider signature contributes with $0.1 \mathrm{~ms}$ to the total verification time but this could be parallelized for multiple signatures. 
Table 1: On-chain storage cost and total verification time for the result section of blocks with 60 contract calling transactions per block (current Ethereum throughput), given different average quorum sizes and number of state changes per transaction.

\begin{tabular}{rrr|rr} 
Tx/Block & Changes/Tx & Quorum Size & Storage & Verification \\
\hline 60 & 4 & 5 & $35 \mathrm{kB}$ & $0.03 \mathrm{~s}$ \\
60 & 10 & 5 & $58 \mathrm{kB}$ & $0.03 \mathrm{~s}$ \\
60 & 25 & 10 & $134 \mathrm{kB}$ & $0.06 \mathrm{~s}$ \\
60 & 50 & 10 & $230 \mathrm{kB}$ & $0.06 \mathrm{~s}$ \\
60 & 100 & 10 & $422 \mathrm{kB}$ & $0.06 \mathrm{~s}$ \\
60 & 200 & 20 & $845 \mathrm{kB}$ & $0.12 \mathrm{~s}$
\end{tabular}

Table 1 shows some examples for block verification time. Even for large quorum sizes of 20 service providers and with an average number of 200 state changes per transaction, total verification cost is small at 0.12 seconds per block.

Storage cost. The second performance metric that we evaluate is storage, measured as the on-chain storage due to execution results. Execution result storage scales linearly with the number of state changes. For each updated 32 byte word, 64 bytes are needed (32 bytes for the location, 32 for the new value). In addition we need to store an ECDSA signature of 64 bytes for each member of the quorum. Note that the storage cost is independent of the execution time, i.e. even long-running contracts may only make very few state changes.

Table 1 shows examples for on-chain storage costs given different average number of storage changes per transaction and different quorum sizes. We assume the current throughput of Ethereum in terms of contract calling transactions per block (60). Depending on the number of state changes and size of quorum, the storage overhead ranges from tens to hundreds of kilobytes. Currently, Ethereum has an average of 4 state changes per transaction, i.e. for similar contracts, the result section would add an overhead of $35 \mathrm{kB}$ to each block, effectively doubling the block size. With a larger number of state changes, the overhead grows linearly.

Note that, while block size increases have been much debated in the community for their implications on security, the largest issue stemming from increased block sizes is increased verification time (caused by execution). ACE addresses exactly this problem by moving execution away from miners. Block propagation time is a smaller issue than often assumed [14].

In addition, new, fast block propagation protocols are being developed with the claim that $1 \mathrm{MB}$ blocks could be propagated to the majority of the Bitcoin network within 1.9 seconds given connection speeds of 56Mbps [18], thus making it feasible to support even contracts with a large number of state changes (e.g. 200) in ACE.

Communication cost. The primary communication cost of ACE is that it may increase block size as described above. All other messages involved in the system have no significant cost.

Latency. Since the transactions are first ordered in a block, then executed and finally the results are included in another block, the latency for results to be committed is at least two blocks instead of one as in Ethereum. However, this is a small overhead compared to the latency usually required for transactions to be considered final in PoW blockchains.

\section{DISCUSSION}

Incentives. Naturally, ACE requires incentives for service providers to participate in the system. This can be easily provided using the gas model from Ethereum. Miners could receive a simple per-byte fee for the inclusion of transactions and the fees for contract execution (based on the used gas) could go to the service providers who executed the contract. Since fewer participants need to execute each contract, the gas price can be expected to be significantly lower than in Ethereum.

Service provider changes. For a long running smart contract, it may be desirable to have the ability to update the set of service providers. For example, one may want to replace a service provider that has become permanently unresponsive. One possible approach is that the deployed smart contract code includes a contract-specific service provider update policy that defines the conditions under which the service providers may be updated after the initial contract deployment.

Signature aggregation. For large executing sets (e.g. 20 or more service providers), the required result signatures increase the the block size and verification time. If large executing sets are used, the block size and the verification time can be reduced by using an aggregate signature scheme such as BLS signatures [4]. The client could collect individual result signatures from $q$ out of $n$ service providers and then aggregate them together before broadcasting the results to the miners. Such optimization is beneficial only for large execution sets, because the verification time of aggregate signatures is high compared to standard signatures like ECDSA.

ACE on top of Ethereum. In principle, ACE could be implemented as a smart contract on top of Ethereum. However, this would require all verification components to run in a smart contract in the Ethereum Virtual Machine instead of natively. Since many operations are costly (in terms of gas) to implement in an Ethereum smart contract (e.g. handling of the queues for verification), the throughput would be very limited.

Further related work. Plasma [26] is another approach for scaling on top of Ethereum by introducing so-called child-chains, which run their own consensus mechanism and process transactions for a specific purpose. This requires explicit transfer of assets between the main chain and a child-chain and complicates interaction between child-chains. Solutions [11,34] parallelize smart contract execution for multiple cores on a single node. In such solutions every node still needs to execute every smart contract call.

\section{CONCLUSION}

Permissionless smart contract platforms like Ethereum are often expected to enable arbitrary financial applications. However, in practice such systems impose heavy restrictions on the types of computations that can be implemented. In this paper, we have described a novel system called ACE that enables several orders of magnitude more complex contracts using off-chain execution by appointed service providers. The key technical ingredient of our solution is a concurrency control protocol that allows contracts to call each other across service provider boundaries but does not require that all service providers must mutually trust each other. 


\section{REFERENCES}

[1] 2016. Solidity. https://solidity.readthedocs.io.

[2] Mustafa Al-Bassam, Alberto Sonnino, Shehar Bano, Dave Hrycyszyn, and George Danezis. 2018. Chainspace: A sharded smart contracts platform. Symposium on Network and Distributed Systems Security (NDSS) (2018).

[3] Philip A Bernstein, Vassos Hadzilacos, and Nathan Goodman. 1987. Concurrency control and recovery in database systems. (1987).

[4] Dan Boneh, Ben Lynn, and Hovav Shacham. 2001. Short signatures from the Weil pairing. In International Conference on the Theory and Application of Cryptology and Information Security. Springer, 514-532.

[5] Ferdinand Brasser, Urs Müller, Alexandra Dmitrienko, Kari Kostiainen, Srdjan Capkun, and Ahmad-Reza Sadeghi. 2017. Software Grand Exposure: SGX Cache Attacks Are Practical. In WOOT. USENIX.

[6] Jo Van Bulck, Marina Minkin, Ofir Weisse, Daniel Genkin, Baris Kasikci, Frank Piessens, Mark Silberstein, Thomas F. Wenisch, Yuval Yarom, and Raoul Strackx 2018. Foreshadow: Extracting the Keys to the Intel SGX Kingdom with Transient Out-of-Order Execution. In USENIX Security. USENIX Association, Baltimore, MD. https://www.usenix.org/conference/usenixsecurity18/presentation/bulck

[7] Guoxing Chen, Sanchuan Chen, Yuan Xiao, Yinqian Zhang, Zhiqiang Lin and Ten H. Lai. 2018. SgxPectre Attacks: Leaking Enclave Secrets via Speculative Execution. CoRR abs/1802.09085 (2018). arXiv:1802.09085 http://arxiv.org/abs/1802.09085

[8] Raymond Cheng, Fan Zhang, Jernej Kos, Warren He, Nicholas Hynes, Noah Johnson, Ari Juels, Andrew Miller, and Dawn Song. 2018. Ekiden: A Platform for Confidentiality-Preserving, Trustworthy, and Performant Smart Contract Execution. arXiv preprint arXiv:1804.05141 (2018).

[9] James C Corbett, Jeffrey Dean, Michael Epstein, Andrew Fikes, Christopher Frost, Jeffrey John Furman, Sanjay Ghemawat, Andrey Gubarev, Christopher Heiser, Peter Hochschild, et al. 2013. Spanner: Google's globally distributed database. ACM Transactions on Computer Systems (TOCS) 31, 3 (2013), 8

[10] Sourav Das, Vinay Joseph Ribeiro, and Abhijeet Anand. 2018. YODA: Enabling computationally intensive contracts on blockchains with Byzantine and Selfish nodes. arXiv preprint arXiv:1811.03265 (2018).

[11] Thomas Dickerson, Paul Gazzillo, Maurice Herlihy, and Eric Koskinen. 2017 Adding concurrency to smart contracts. In Proceedings of the ACM Symposium on Principles of Distributed Computing. ACM, 303-312.

[12] Kapali P. Eswaran, Jim N Gray, Raymond A. Lorie, and Irving L. Traiger. 1976 The notions of consistency and predicate locks in a database system. Commun. ACM 19, 11 (1976), 624-633.

[13] Daniel Genkin, Lev Pachmanov, Itamar Pipman, Adi Shamir, and Eran Tromer 2016. Physical key extraction attacks on PCs. Commun. ACM 59, 6 (2016).

[14] Arthur Gervais, Ghassan O Karame, Karl Wüst, Vasileios Glykantzis, Hubert Ritzdorf, and Srdjan Capkun. 2016. On the security and performance of proof of work blockchains. In Proceedings of the 2016 ACM SIGSAC conference on computer and communications security. ACM, 3-16.

[15] Johannes Götzfried, Moritz Eckert, Sebastian Schinzel, and Tilo Müller. 2017. Cache attacks on Intel SGX. In European Workshop on Systems Security. ACM.

[16] Ethan Heilman, Alison Kendler, Aviv Zohar, and Sharon Goldberg. 2015. Eclipse Attacks on Bitcoin's Peer-to-Peer Network. In Proceedings of the 24th USENIX Security Symposium (USENIX Security).
[17] Harry Kalodner, Steven Goldfeder, Xiaoqi Chen, S Matthew Weinberg, and Edward W Felten. 2018. Arbitrum: Scalable, private smart contracts. In Proceedings of the 27th USENIX Conference on Security Symposium. USENIX Association, 1353-1370.

[18] Uri Klarman, Soumya Basu, Aleksandar Kuzmanovic, and Emin Gün Sirer. [n.d.]. bloXroute: A Scalable Trustless Blockchain Distribution Network WHITEPAPER. ([n.d.]).

[19] Eleftherios Kokoris-Kogias, Philipp Jovanovic, Linus Gasser, Nicolas Gailly, Ewa Syta, and Bryan Ford. 2018. Omniledger: A secure, scale-out, decentralized ledger via sharding. In 2018 IEEE Symposium on Security and Privacy (SP). IEEE, 583-598.

[20] Hsiang-Tsung Kung and John T Robinson. 1981. On optimistic methods for concurrency control. ACM Transactions on Database Systems (TODS) 6, 2 (1981), 213-226.

[21] Loi Luu, Jason Teutsch, Raghav Kulkarni, and Prateek Saxena. 2015. Demystifying incentives in the consensus computer. In Proceedings of the ACM Conference on Computer and Communications Security (CCS). ACM.

[22] Ahmad Moghimi, Gorka Irazoqui, and Thomas Eisenbarth. 2017. Cachezoom: How SGX amplifies the power of cache attacks. In Cryptographic Hardware and Embedded Systems. Springer.

[23] Satoshi Nakamoto. 2008. Bitcoin: A peer-to-peer electronic cash system. (2008).

[24] Christos H. Papadimitriou. 1979. The Serializability of Concurrent Database Updates. 7. ACM 26, 4 (Oct. 1979), 631-653. https://doi.org/10.1145/322154.322158

[25] Rafael Pass and Elaine Shi. 2017. Rethinking large-scale consensus. In 2017 IEEE 30th Computer Security Foundations Symposium (CSF). IEEE, 115-129.

[26] Joseph Poon and Vitalik Buterin. 2017. Plasma: Scalable autonomous smart contracts. White paper (2017)

[27] Kun Ren, Alexander Thomson, and Daniel J Abadi. 2014. An evaluation of the advantages and disadvantages of deterministic database systems. Proceedings of the VLDB Endowment 7, 10 (2014), 821-832.

[28] Hubert Ritzdorf, Karl Wüst, Arthur Gervais, Guillaume Felley, et al. 2018. TLS-N: Non-repudiation over TLS Enabling Ubiquitous Content Signing. In Network and Distributed System Security Symposium (NDSS).

[29] Ewa Syta, Philipp Jovanovic, Eleftherios Kokoris Kogias, Nicolas Gailly, Linus Gasser, Ismail Khoffi, Michael J Fischer, and Bryan Ford. 2017. Scalable bias-resistant distributed randomness. In 2017 IEEE Symposium on Security and Privacy (SP). Ieee, 444-460.

[30] Alexander Thomson, Thaddeus Diamond, Shu-Chun Weng, Kun Ren, Philip Shao, and Daniel J Abadi. 2012. Calvin: fast distributed transactions for partitioned database systems. In Proceedings of the 2012 ACM SIGMOD International Conference on Management of Data. ACM, 1-12.

[31] Gavin Wood. 2014. Ethereum: A secure decentralised generalised transaction ledger. (2014)

[32] Gavin Wood. 2018. Ethereum: A secure decentralised generalised transaction ledger BYZANTIUM VERSION (e94ebda - 2018-06-05). https://ethereum.github.io/yellowpaper/paper.pdf. Accessed: 2018-08-30.

[33] Yuanzhong Xu, Weidong Cui, and Marcus Peinado. 2015. Controlled-channel attacks: Deterministic side channels for untrusted operating systems. In SP. IEEE.

[34] An Zhang and Kunlong Zhang. 2018. Enabling Concurrency on Smart Contracts Using Multiversion Ordering. In Asia-Pacific Web (APWeb) and Web-Age Information Management (WAIM) foint International Conference on Web and Big Data. Springer, 425-439. 


\section{A BACKGROUND}

In this appendix we provide background information on smart contracts and concurrency control.

\section{A.1 Ethereum Smart Contracts}

The Ethereum protocol [32] defines a system for a decentralized state machine by using the blockchain paradigm. It allows participating nodes to write and execute contracts that are a collection of functions and global storage variables. Whenever a function is called, the system's state may change. This can be achieved through transactions that define the target contract and the transaction sender. Each block defines a specific system state and the transactions it contains represent the transition from the state of the block's predecessor to its current state.

The Ethereum state consists of two types of account objects: external accounts controlled by a private key and contract accounts controlled by the contract code. An external account can send messages to other accounts in the form of transactions. If a contract account receives a message call, its code is executed which might read or write to the account's state, send messages to other contracts or create new contracts.

Miners collect transactions from a peer-to-peer network and add them to a new block that is distributed back to all miners who execute all included transactions to verify them. To keep verification fast, every used resource (computation, storage) defines a price, measured in gas, and every transaction has a gas limit. The gas used during execution is subtracted from the account balance of the transaction sender and added to the block miner's account.

Ethereum contracts can be written in high-level languages, such as Solidity [1], that are compiled into a low-level, stack-based, Turing-complete language called EVM (Ethereum Virtual Machine) Code. Executing the EVM Code is handled by an EVM interpreter.

Ethereum's trust assumptions follow the general security model of PoW blockchains [14]. It is assumed that no malicious entity controls a majority of the mining power and message dissemination works sufficiently well (e.g., eclipse attacks are not possible [16]). For contracts, Ethereum's trust model is contract-specific, i.e., users can freely choose which contracts they decide to trust. For example, if a user decides to send money to a particular smart contract, then with this action the user implicitly agrees with the conditions and the logic specified in that contract's code. A user that decides to trust one contract does not have to trust other contracts that run in the same system.

\section{A.2 Concurrency Control}

Distributed database systems process transactions using multiple database servers or partitions [9]. Transactions consist of multiple operations (reads and writes) and the execution order of these operations is called schedule. If two transactions access the same resource simultaneously, they may conflict. To ensure safe concurrent processing, one usually wants to achieve strict serializability [24]. Strict serializability is a strong level of isolation that ensures that the outcome of transactions executed in parallel is equal to one in which the transactions had been executed atomically and sequentially in the order apparent to an external observer.

Several concurrency protocols are known. Two-phase locking is a classical solution that enables serializable schedules [12]. To ensure atomicity and durability, the transaction results must be committed either on all or none of the involved resources that may cross partition boundaries. The involved partitions run a distributed commit protocol that requires multiple rounds of communication (two rounds are required for crash faults and three rounds for Byzantine faults). Such distributed commit protocol is typically considered the primary cost of distributed transaction processing.

Another classical concurrency control solution are optimistic protocols [20]. In such systems, distributed transactions are first executed without locking and afterwards the transaction managers examine if conflicts took place. If this is the case, all execution results must be rolled back which incurs a significant cost. If none of the executed operations were in conflict, the execution results can be committed (using a commit protocol).

Recently, deterministic concurrency control has been proposed as an alternative [27]. In deterministic systems, transactions are first ordered by a (centralized or distributed) sequencing layer followed by a simple and deterministic locking protocol. Executions must follow this order regardless of possible crashes. The primary benefit of deterministic concurrency control solutions is that they avoid the high cost of multi-round distributed commit protocols.

A transaction schedule is called recoverable if abort of one transaction never leaves other transactions in inconsistent state. A transaction schedule is called cascading or cascadeless recoverable depending if the abort of one transaction requires rolling back other (not yet committed) transactions or not. 